\title{
Absence of Dirac cones in monolayer silicene and multilayer Si films on $\operatorname{Ag}(111)$
}

S. K. Mahatha ${ }^{1, *}$, P. Moras ${ }^{1}$, P. M. Sheverdyaeva ${ }^{1}$, V. Bellini' ${ }^{2}$, T. O. Menteş ${ }^{3}$, A. Locatelli ${ }^{3}$, R. Flammini $^{4}$, K. Horn ${ }^{5}$ and C. Carbone ${ }^{1}$

${ }^{1}$ Istituto di Struttura della Materia, Consiglio Nazionale delle Ricerche, I-34149 Trieste, Italy

${ }^{2}$ Istituto di Nanoscienze, Consiglio Nazionale delle Ricerche, I-41125 Modena, Italy

${ }^{3}$ Elettra-Sincrotrone Trieste S.C.p.A, SS 14, Km 163,5, I-34149, Trieste, Italy

${ }^{4}$ Istituto di Struttura della Materia, Consiglio Nazionale delle Ricerche, I-00133 Roma, Italy

${ }^{5}$ Fritz-Haber-Institut der Max-Planck-Gesellschaft, Faradayweg 4-6, D-14195 Berlin, Germany

\begin{abstract}
Monolayer silicene and multilayer silicon films on $\operatorname{Ag}(111)$ have been the subject of many investigations within the last few years. For both systems, photoemission data have been interpreted in terms of linearly dispersing bands giving rise to the characteristic Dirac cone features, similar to graphene. Here we demonstrate, on the basis of angle-resolved valence band and core level photoemission data that this assignment is not correct. The bands previously attributed to states with Dirac fermion character are shown to derive from $\mathrm{Ag}(111)$ interface and bulk states in silicene monolayer and from the well-known $\mathrm{Ag}-(\sqrt{3} \times \sqrt{3}) \mathrm{R} 30^{\circ}-\mathrm{Si}(111)$ structure in Si multilayers. These results question the validity of the claim that graphene-like silicene and silicene multilayers are in fact formed on $\operatorname{Ag}(111)$.
\end{abstract}

Keywords: 2D materials, silicene, multilayer silicene, electronic structure, angle-resolved photoemission

*corresponding author: sanjoymahatha@gmail.com 


\section{INTRODUCTION}

The discovery of graphene with its rich physical properties has sparked tremendous interest towards the study of other two-dimensional (2D) materials with honeycomb structure. ${ }^{1}$ It was believed that a free-standing 2D sheet with long range order cannot exist in nature due to the thermal fluctuations, inducing melting of the 2D sheet into a three-dimensional (3D) material. ${ }^{2}$ However, graphene has been shown to be stable as a 2D material and has emerged with a different physics that connects quantum electrodynamics and condensed matter physics. ${ }^{3}$ This motivated to explore the electronic structure of other group IV elements, in the form of either free-standing or supported monolayers. In this context, silicene, a 2D honeycomb lattice of silicon, has been studied extensively in the last few years.

Silicene $^{4}$ is theoretically predicted to be stable as a free standing monolayer in a low buckled geometry and is expected to display Dirac cones like graphene ${ }^{5-8}$ with a comparable group velocity, ${ }^{9}$ and thus may form the basis for a multitude of applications. ${ }^{10}$ While free-standing silicene has not so far been realized, Si deposition on different surfaces has been pursued as an alternative method to synthesize silicene. The formation of honeycomb silicene monolayers (ML) has been reported on $\operatorname{Ag}(111)^{11-21}, \mathrm{ZrB}_{2}(0001),{ }^{22,23}$ and $\operatorname{Ir}(111)^{24}$ surfaces. Si films on $\operatorname{Ag}(111)^{13-21,25}$ have been studied most extensively. In the monolayer and sub-monolayer range, $\mathrm{Si}^{26}$ forms a mixture of $(4 \times 4)$, $(\sqrt{13} \times \sqrt{13}) \mathrm{R} 13.9^{\circ}$ and $(2 \sqrt{3} \times 2 \sqrt{3}) \mathrm{R} 30^{\circ}$ (w.r.t. $\mathrm{Ag}(111)$ unit cell) reconstructed phases, depending on the growth parameters. Such structures present different atomic buckling, arising from the registry relations between silicene and the Ag lattice.

A main open question in silicene research concerns the strength of interfacial interactions and how silicene electronic structure is affected by them. In particular, it has been debated whether the characteristic Dirac cones formed by the $\pi$-symmetry bands, ${ }^{16}$ showing linear dispersion similarly to graphene, are preserved for the case of supported silicene. ${ }^{27}$ Even though we show in the $\operatorname{Ag}(111)$ do not exhibit such features in their band structure, we will adhere to the usage of "silicene" for these structures in line with the literature, but this point should be kept in mind in the discussion.

The most simple and best characterized monolayer silicene phase on $\operatorname{Ag}(111)$ shows a $(4 \times 4)$ structure. Angle-resolved photoemission spectroscopy (ARPES) on this phase allowed a substratemodified Dirac cone of $\pi$-band at the $\overline{\mathrm{K}}_{\mathrm{Si}}$ point to be identified as opening a gap at $0.3 \mathrm{eV}$ below Fermi energy $\left(\mathrm{E}_{\mathrm{F}}\right) .{ }^{16} \mathrm{~A}$ similar $\pi$-band Dirac cone feature was also claimed to be found at the $\overline{\mathrm{M}}_{\mathrm{Ag}}$ point of the Ag surface ${ }^{28}$ and at the equivalent points of the $(4 \times 4)$-silicene Brillouin zone centers. However, the absence of Landau levels in scanning tunneling spectroscopy (STS) measurements under high magnetic field ${ }^{21}$ and theoretical calculations ${ }^{21,29-31}$ questioned this interpretation. In 
addition to the monolayer case, ARPES studies on multilayer Si films on $\operatorname{Ag}(111)$ assigned certain spectral features observed near $\mathrm{E}_{\mathrm{F}}$ to the presence of Dirac cones. This claim was taken as evidence for a weak interaction between the Si planes, and led to the description of the film as multilayer silicene. ${ }^{32-35}$ Such Si films, in excess of one ML, present a $(\sqrt{3} \times \sqrt{3}) \mathrm{R} 30^{\circ}$ reconstructed surface in low energy electron diffraction (LEED) with respect to an ideally flat silicene lattice. ${ }^{15,17-19,32}$ Theoretical studies have proposed that the multilayer structure consists of a stacking of dumbbell patterned Si layers with a $(\sqrt{3} \times \sqrt{3}) \mathrm{R} 30^{\circ}$ periodicity, ${ }^{36}$ with stronger in-plane than out-of plane bonding. However, the calculated band structure presents a band gap larger than in diamond-like Si, in contrast with the ARPES results. ${ }^{32}$ Moreover, scanning tunneling microscopy (STM), STS, atomic force microscopy (AFM), core level photoemission, LEED-IV and Raman measurements, indicate that the Si films has a Ag terminated diamond-like Si(111) structure. ${ }^{37-44}$ In order to dispute the ARPES results that were in conflict with the silicene multilayers, it was suggested that multilayer silicene on $\mathrm{Ag}(111)$ might only be fabricated in a narrow temperature range. ${ }^{45}$

To assess whether silicene and Si multilayers display Dirac cones, as claimed by other photoemission investigations, we performed extensive ARPES investigations, corroborated by LEED, low-energy electron microscopy (LEEM), core level photoemission measurements as well as band structure calculation analysis. Our experiments allowed us to clarify unambiguously the nature of $\mathrm{Si}-\mathrm{Ag}$ interfacial interactions and their relation to the silicene electronic structure. ARPES on $(4 \times 4)$ silicene monolayer highlights the effects of $\mathrm{Si}-\mathrm{Ag}$ interaction at the interface. In the following, we will show that, due to hybridization with the Ag states, the silicene states with $\pi$ symmetry lose their 2D character, resulting in the disruption of the Dirac cone. For Si multilayers grown on $\operatorname{Ag}(111)$ we find that the band structure of the films closely corresponds to the one of the $(\sqrt{3} \times \sqrt{3}) \mathrm{R} 30^{\circ}-\mathrm{Ag}$ terminated $\mathrm{Si}(111)$ surface, in line with the conclusions of structural investigations..

\section{EXPERIMENTAL METHODS}

The clean $\mathrm{Ag}(111)$ surface was prepared by cycles of $\mathrm{Ar}^{+}$sputtering and UHV annealing at $\sim 830 \mathrm{~K}$. The order and cleanliness of the surface was confirmed by LEED. Si was deposited by resistive heating of a $\mathrm{Si}$ wafer at a rate of $0.01 \mathrm{ML} / \mathrm{min}$ on $\mathrm{Ag}(111)$, in accordance to the rate used in other studies. $^{12,13,17,18,21}$ Here one monolayer of $\mathrm{Si}$ is defined as the quantity corresponding to the completion of the Si wetting layer at $\sim 500 \mathrm{~K}$, according to Ref. [26]. Multilayer Si films were grown at $\sim 470 \mathrm{~K}$. The $(\sqrt{3} \times \sqrt{3}) \mathrm{R} 30^{\circ}-\mathrm{Ag} / \mathrm{Si}(111)$ surface was prepared by deposition of $\sim 1$ monolayer of $\mathrm{Ag}$ onto clean $\mathrm{Si}(111)-(7 \times 7)$ surface at room temperature, followed by annealing to $\sim 870 \mathrm{~K}$. 
LEED and LEEM measurements were carried out using the SPELEEM (Spectroscopic PhotoEmission and Low Energy Electron Microscope) instrument at the Nanospectroscopy beamline of the Elettra synchrotron. ${ }^{46}$ The electron source is an $\mathrm{LaB}_{6}$ cathode, with $0.4 \mathrm{eV}$ energy spread. The transfer width of the instrument is about $130 \AA$. In the diffraction mode, the incident electron beam is limited to a micron-sized area by using an illumination aperture. In LEEM imaging, the lateral resolution is about $10 \mathrm{~nm}$. By inserting an angle-selecting aperture in the diffraction plane, dark-field imaging (df-LEEM) can be performed in order to map out structural domains characterized by distinct LEED patterns.

The ARPES and core level photoemission spectroscopy measurements reported here were performed at the VUV-Photoemission beamline of the Elettra synchrotron using a Scienta R4000 electron spectrometer. In these measurements the spectrometer was placed at an angle of $45^{\circ}$ with respect to the direction of the p-polarized photon beam. Photoelectrons were collected within the light scattering plane. All photoemission measurements were performed at room temperature with an angular resolution better than $0.3^{\circ}$ and an energy resolution of $30 \mathrm{meV}$. Core level data were acquired at photon energy of $150 \mathrm{eV}$ for the $\mathrm{Si} 2 p$ and $430 \mathrm{eV}$ for the $\mathrm{Ag} 3 d$ core level. ARPES spectra of monolayer and multilayer Si were measured using $126 \mathrm{eV}$ and $135 \mathrm{eV}$ photon energy. The binding energy were calibrated by determining $E_{\mathrm{F}}$ on a clean $\operatorname{Ag}(111)$ surface. Ab-initio band structure calculations were performed using the VASP code $^{47}$ with the GGA-PBE exchangecorrelation potential $^{48}$ and including van der Waals interactions in the semi-empirical method of Grimme. $^{49}$

\section{RESULTS AND DISCUSSION}

\section{III.A Growth characterization: $(4 \times 4)$ and $(\sqrt{13} \times \sqrt{13})$ R13.9 ${ }^{\circ}$ structures}

Silicene on $\operatorname{Ag}(111)$ presents a mixed phase structure, with proportions depending on coverage and deposition temperature. ${ }^{26}$ In LEED, all these structures exhibit the same lattice constant, but may be rotated at different angles with respect to the $\operatorname{Ag}(111)$ substrate. Fig.1 shows the evolution of the LEED pattern taken at an electron energy of $31 \mathrm{eV}$ as a function increasing $\mathrm{Si}$ coverage at $500 \mathrm{~K}$. As can be seen, the initial $(1 \times 1)$ pattern of the clean Ag substrate quickly evolves into a superposition of several hexagonal patterns closer to the specular (00) spot, matching the lattice unit of $\mathrm{Si}$. At $1 \mathrm{ML} \mathrm{Si}$ coverage, two prominent structures are clearly visible: a $(4 \times 4)$ pattern aligned with the $\operatorname{Ag}(1 \times 1)$ unit vectors, and a $(\sqrt{13} \times \sqrt{13}) \mathrm{R} 13.9^{\circ}$ pattern with two equivalent domains symmetrically placed close to the $(4 \times 4)$ spot. The angle between the $(\sqrt{13} \times \sqrt{13})$ R13.9 and the $(4 \times 4)$ lattices is about $5^{\circ}$. At higher temperature, another rotational silicene domain, $(2 \sqrt{3} \times 2 \sqrt{3}$ ), becomes visible (not shown in the figure). Importantly, the surface is never uniformly 
covered with a single silicene phase at any temperature or coverage. Upon completion of the silicon monolayer, additional spots appear at locations corresponding to the $(\sqrt{3} \times \sqrt{3}) \mathrm{R} 30^{\circ}$ structure in the basis of the Si lattice. The new spots are marked in Fig.1(d) by a dashed hexagon. A close inspection of the azimuthal profile of the new pattern clearly reveals the emergence of this $(\sqrt{3} \times \sqrt{3}) \mathrm{R} 30^{\circ}$ structure, corresponding to each rotational Si domain $\left((4 \times 4)\right.$ and $\left.(\sqrt{13} \times \sqrt{13}) \mathrm{R} 13.9^{\circ}\right)$. The $(\sqrt{3} \times \sqrt{3}) \mathrm{R} 30^{\circ}$ structure is identified with two layer silicene on $\mathrm{Ag}(111){ }^{26}$ A detailed structural study on the growth of different phases of silicene on $\operatorname{Ag}(111)$ as a function of the deposition temperature and coverage using LEED, LEEM has been reported elsewhere. ${ }^{26}$

Fig. 2(a) displays the LEED pattern of silicene on $\mathrm{Ag}(111)$ grown at $~ 500 \mathrm{~K}$ comprising intense $(4 \times 4)$ and relatively broad and weaker $(\sqrt{13} \times \sqrt{13}) \mathrm{R} 13.9^{\circ}$ spots, due to azimuthally disordered domains. Fig.2(b) shows maps different Si rotational domains. The LEEM image on the right panel highlights the $(4 \times 4)$ and $(\sqrt{13} \times \sqrt{13}) \mathrm{R} 13.9^{\circ}$ domains, which cover almost the entire surface. Note that the two rotational $(\sqrt{13} \times \sqrt{13})$ R $13.9^{\circ}$ domains are seen with the same contrast as the figure displays the sum of two dark-field images using the LEED spots of the two $(\sqrt{13} \times \sqrt{13}) \mathrm{R} 13.9^{\circ}$ rotational domains. The domain size is of the order of $200 \mathrm{~nm}$ regardless of the step morphology of the substrate. The limited extent of domains is likely due to the high nucleation density of silicene islands at the temperatures considered. The area coverages of the two rotational $(\sqrt{13} \times \sqrt{13})$ R $13.9^{\circ}$ domains are both equal to about $30 \%$. Fig. 2(c) shows the surface Brillouin zone of $\operatorname{Ag}(111)$, of an ideal $(1 \times 1)$ silicene and a $(4 \times 4)$ silicene structure, and of the $(\sqrt{3} \times \sqrt{3}) \operatorname{R} 30^{\circ}$ multilayer Si structure. It is important to note that for $(4 \times 4)$ silicene, the $\overline{\mathrm{K}}_{\mathrm{Si}}$ and $\overline{\mathrm{M}}_{\mathrm{Ag}}$ points are equivalent as both symmetry points fold into $\bar{\Gamma}_{\mathrm{Si}}^{4}$ point of $(4 \times 4)$ structure while, for multilayer $\mathrm{Si}$ film on $\operatorname{Ag}(111) \overline{\mathrm{K}}_{\mathrm{Si}}$ point is a $\bar{\Gamma}_{\mathrm{Si}}^{\sqrt{3}}$ point in the second Brillouin zone of $(\sqrt{3} \times \sqrt{3}) \mathrm{R} 30^{\circ}$ structure.

\section{III.B Band structure of a silicene monolayer.}

ARPES studies were performed on silicene grown at $\sim 500 \mathrm{~K}$ to achieve a flat silicene layer on $\operatorname{Ag}(111)$, where the $(4 \times 4)$ - phase is present with $(\sqrt{13} \times \sqrt{13}) \mathrm{R} 13.9^{\circ}$ phase. Fig. 3 (a) displays the theoretical electronic band structure of free-standing and unreconstructed $(1 \times 1)$ silicene, displaying bands of $\pi$ - (orange line) and $\sigma$-symmetry (green line) character. Fig. 3(b) shows the experimental band structure of mixed $(4 \times 4)$ and $(\sqrt{13} \times \sqrt{13}) \mathrm{R} 13.9^{\circ}$ phase of silicene grown on $\operatorname{Ag}(111)$. Comparing the ARPES results (Fig. 3(b)) with the calculated band structure (Fig. 3(a)), we identify the deeper Si $\sigma$-band with a minima at $\sim 12.5 \mathrm{eV}$ and the continuation of the same band with a maximum around $1.3 \mathrm{eV}$ binding energy. Indeed the energy dispersion of this band precisely corresponds to the one of $\sigma$-band. This band is better visible in the second Brillouin zone of the 
(1×1)- flat silicene (as shown in Fig.3(b) with a dashed green line) along with weaker replicas at corresponding wave vectors of the $(4 \times 4)$ and $(\sqrt{13} \times \sqrt{13}) \mathrm{R} 13.9^{\circ}$ cells. Consistent with the LEED pattern, photoemission bands corresponding to $(\sqrt{13} \times \sqrt{13}) \mathrm{R} 13.9^{\circ}$ domains rotated by $5.2^{\circ}$ with respect to $(4 \times 4)$ have also been observed with much weaker and diffuse intensity (not shown). Similar to the case of the $(4 \times 4)$ structures, the bands at 1.3 binding energy have been attributed to the $\sigma$ bands of the $(\sqrt{13} \times \sqrt{13}) \mathrm{R} 13.9^{\circ}$ silicene phases. Along with these two Si- $\sigma$ bands, very intense localized $\mathrm{Ag} 4 d$ bands and bands with free-electron like parabolic dispersion crossing $\mathrm{E}_{\mathrm{F}}$ are also visible. Earlier ARPES studies identified this highly dispersing states as Dirac states arising from the $\pi$ band of silicene.

However, a closer inspection of these bands near the Fermi level along $\bar{\Gamma}_{\mathrm{Ag}}-\overline{\mathrm{K}}_{\mathrm{Ag}}$ direction in silicene on $\operatorname{Ag}(111)$ (Fig. 4(a,b)), demonstrates that they exist and exhibit identical dispersion as in clean $\mathrm{Ag}$ (Fig. 4(c,d)), though with a different $-\mathrm{k}_{\|}$vs $+\mathrm{k}_{\|}$intensity. ${ }^{50}$ This leads us to conclude that the bands at $\overline{\mathrm{K}}_{\mathrm{Si}}$ near $\mathrm{E}_{\mathrm{F}}$ are the $\mathrm{Ag} s p$-bulk bands. Also the data in Fig. 4(a-d), measured with good statistics, show no evidence for a gap opening at the $\overline{\mathrm{K}}_{\mathrm{Si}}$ point $\left(1.1 \AA^{-1}\right)$, in contrast to a previous study. ${ }^{16}$ Another work on monolayer silicene identified a band near $\overline{\mathrm{M}}_{\mathrm{Ag}}$ point as silicene $\pi$-band with a $\pi$ - $\pi^{*}$ gap opening of $0.33 \mathrm{eV} \cdot{ }^{28}$ Due to the equivalence of the $\overline{\mathrm{M}}_{\mathrm{Ag}}$ point and the $\overline{\mathrm{K}}_{\mathrm{Si}}$ point of $(4 \times 4)$ cell (as shown in Fig. 2(c)) this band was also attributed to the $\pi$-state with a Diraclike dispersion. However, a more detailed investigation of this ARPES feature disproves this assignment. Fig. 4(e, g) and (f, g) presents spectra of silicene on $\operatorname{Ag}(111)$ and clean $\operatorname{Ag}(111)$ respectively, in the vicinity of the $\overline{\mathrm{M}}_{\mathrm{Ag}}$ point along two orthogonal directions. A (I) band with a maximum at $\sim 0.35 \pm 0.05 \mathrm{eV}$ at the $\overline{\mathrm{M}}_{\mathrm{Ag}}$ point, close to the bulk Ag $s p$ band is visible (Fig. 4(e) and (f)). The (I) band displays a energy maximum along the $\bar{\Gamma}_{\mathrm{Ag}}-\overline{\mathrm{M}}_{\mathrm{Ag}}-\bar{\Gamma}_{\mathrm{Ag}}$ direction and a minimum in the perpendicular $\overline{\mathrm{K}}_{\mathrm{Ag}}-\overline{\mathrm{M}}_{\mathrm{Ag}}-\overline{\mathrm{K}}_{\mathrm{Ag}}$ direction also reported in Ref. [51]. This indicates that the (I) band has the shape of a saddle (as shown in Fig. 4(i)) rather than a cone.

We find that this band exists only on a silicene-covered $\operatorname{Ag}(111)$ surface and has a 2D character, since it shows no dispersion by varying the photon energy. Through theoretical analysis, ${ }^{50,52}$ this band is assigned to an interface state, split-off from the Ag sp bands, near the edge of the Ag surface-projected bulk bands. Although this interface state is induced by the silicene deposition, it is largely $(\sim 75-80 \%)$ of $\mathrm{Ag}$, $s p$ character, as shown by its dispersion that mimics the one of a free-electron band, folded into the $(4 \times 4)$ cell, and also by its charge density distribution. ${ }^{52}$ It turns out that this silicene induced interface state is strongly localized in the first Ag layer, with a significant density also in the second Ag layer. ${ }^{51}$ In summary, a saddle-shaped band, originated 
from the a Ag derived interface state is formed, but no Dirac cones can be observed in silicene on $\operatorname{Ag}(111)$.

\section{III.C Multilayer Si films with $(\sqrt{3} \times \sqrt{3}) R^{\circ} 30^{\circ}$ surface structure}

Since a multilayer stacked arrangement of silicene was suggested to exist on $\operatorname{Ag}(111)$ in a particular deposition temperature $(470 \mathrm{~K})$ range. We examined the electronic bands structure of multilayer Si film on $\operatorname{Ag}(111)$ by core level photoemission and ARPES and compared it to the one of $(\sqrt{3} \times \sqrt{3}) \mathrm{R} 30^{\circ}-\mathrm{Ag} / \mathrm{Si}(111)$. Fig. 5(a) and (b) display the $\mathrm{Ag} 3 d_{5 / 2}$ and $\mathrm{Si} 2 p$ spectra measured on 7 ML Si film on $\mathrm{Ag}(111)$, respectively. $\mathrm{An} \mathrm{Ag} 3 d_{5 / 2}$ peak, shifted with respect to the one from the substrate, is observed on the $7 \mathrm{ML}$ Si film. The non-exponential dependence of the area under the $\mathrm{Ag} 3 d$ peak as a function of Si coverage (as shown in inset of Fig. 5(a)) provides some hints on the microscopic processes causing the energy shift with an indication towards dewetting, clustering or Ag segregation to the top Si surface. We compare it with the Ag $3 d_{5 / 2}$ data obtained by depositing a very thick film (few tens of $\mathrm{nm}$ ) of Si on $\mathrm{Ag}(111)$ (Fig. 5(c)) and using a mask to cover a portion of the clean Ag surface. The thick Si film on $\mathrm{Ag}(111)$ (purple color) can be recognized in Fig. 5(d). Fig. 5(c) displays the $\mathrm{Ag} 3 d_{5 / 2}$ spectra measured on three different regions: on clean $\mathrm{Ag}$, on the thick Si film, and on the step edge between these two regions. All the Ag $3 d_{5 / 2}$ spectra are plotted with arbitrary intensity scale and each is normalized to the peak intensity. The Ag $3 d_{5 / 2}$ spectra on the $\mathrm{Si} / \mathrm{Ag}$ step edge clearly shows two components separated by $\sim 0.5 \mathrm{eV}$ binding energy, which corresponds to the binding energy for the $\mathrm{Ag}(\sqrt{3} \times \sqrt{3}) \mathrm{R} 30^{\circ}-\mathrm{Ag}$ terminated $\mathrm{Si}(111)$ surface. A simple decomposition approach shows that this component is predominant in the 7 ML Si spectra, indicating Ag segregation. The unshifted component indicates that the Si film is either highly nonuniform or there are small uncovered regions of the substrate. We have grown $(\sqrt{3} \times \sqrt{3}) \mathrm{R} 30^{\circ}-\mathrm{Si}$ films of different thickness and at different temperatures on $\operatorname{Ag}(111)$, which all shows the two components described above, as discussed in detail in Ref. [53]. Further support for the formation of a $(\sqrt{3} \times \sqrt{3}) \mathrm{R} 30^{\circ}-\mathrm{Ag}$ surface on $7 \mathrm{ML} \mathrm{Si}$ on $\mathrm{Ag}(111)$ is provided by the resemblance of the $\mathrm{Si} 2 p$ spectra which show a similar lineshape although significantly broadened as shown in Fig. 5(b) . Hence, the lineshape and binding energy, as well as an intensity analysis ${ }^{53}$ of $\mathrm{Ag} 3 d_{5 / 2}$ and $\mathrm{Si} 2 p$ core level data from the multilayer Si films on $\mathrm{Ag}(111)$ provide evidence for Ag segregation, and an indication for the formation of $(\sqrt{3} \times \sqrt{3}) \mathrm{R} 30^{\circ}-\mathrm{Ag}$ superstructure on the film surface.

Direct support for the formation of a $(\sqrt{3} \times \sqrt{3}) \mathrm{R} 30^{\circ}-\mathrm{Ag} \operatorname{Si}(111)$ structure is provided by the ARPES results. Fig. 6(a, b) compares ARPES data taken on a $\sim 6 \mathrm{ML} \mathrm{Si}$ film on

$\operatorname{Ag}(111)$ and $(\sqrt{3} \times \sqrt{3}) \mathrm{R} 30^{\circ}-\mathrm{Ag}$ terminated $\mathrm{Si}(111)$, along the $\overline{\mathrm{K}}_{\mathrm{Si}}-\bar{\Gamma}_{\mathrm{Si}}-\overline{\mathrm{M}}_{\mathrm{Si}}$ symmetry directions, measured under the same experimental conditions. The ARPES data of the two systems 
show a very similar band structure. At $\bar{\Gamma}_{\mathrm{Si}}$ the three downward dispersing bands are visible in both the surfaces. The inner two bands correspond to those measured in previous studies that covered a limited energy and momentum range, which were interpreted as $\pi$ Dirac-bands of multilayer and monolayer silicene. ${ }^{28,32}$ The three bands near $\bar{\Gamma}_{\mathrm{Si}}$ correspond to the ones originating from $s p^{3}$ hybridized Si orbitals, namely: the heavy-hole, light-hole, and split-off bands. ${ }^{54}$ Some minor differences between the $\mathrm{Si}$ thin films to the bulk spectra are likely to reflect finite size and structural distortions.

A nearly-parabolic band close to $\mathrm{E}_{\mathrm{F}}$ is observed in 6 ML Si film data (Fig. 6(a)) but is absent in the $(\sqrt{3} \times \sqrt{3}) \mathrm{R} 30^{\circ}-\mathrm{Ag}$ surface (Fig. 6(b)). This band was assigned to a replica of the $\pi^{*}$ Diracband, which should appear at $\overline{\mathrm{K}}_{\mathrm{Si}}$ in multilayer silicene. ${ }^{28,32} \mathrm{We}$ find that the effective mass $(\mathrm{m} * / \mathrm{m}$ $=0.4$, where $\mathrm{m}$ is the mass of free electron) of this parabolic band closely corresponds to that of clean $\mathrm{Ag}(111)$ Shockley surface state. ${ }^{55}$ It shows a difference in binding energy in comparison with clean $\mathrm{Ag}(111)$ that we assign to p-type doping by the adjacent $\mathrm{Si}$. This suggests that $\mathrm{Ag}$ surface state develops into an interface (I) state inside the $310 \mathrm{meV}$ L-gap of the $\mathrm{Ag}(111)$ surface.

At $\overline{\mathrm{K}}_{\mathrm{Si}}\left(\mathrm{k}_{\|}= \pm 1.1 \AA^{-1}\right)$, which is equivalent to the $\bar{\Gamma}_{\mathrm{Si}}$ point in the second Brillouin zone of the $(\sqrt{3} \times \sqrt{3})$ R $30^{\circ}$ surface, we identify three surface states labeled as S1, S2, and S3 according to Ref. $[56,57]$. These band features are characteristic of $(\sqrt{3} \times \sqrt{3}) \mathrm{R} 30^{\circ}-\mathrm{Ag}$ terminated $\mathrm{Si}(111)$. The parabolic band at $\mathrm{E}_{\mathrm{F}}(\mathrm{S} 1)$ was also interpreted as a $\pi^{*}$ Dirac-band of multilayer silicene, ${ }^{32,33}$ since due to its position in $\mathrm{k}$-space it could be a replica of the parabolic band at $\bar{\Gamma}_{\mathrm{Si}}$ in the first Brillouin zone. However the bands at $\bar{\Gamma}_{\mathrm{Si}}\left(\mathrm{k}_{\|}=0 \AA^{-1}\right)$ and $\overline{\mathrm{K}}_{\mathrm{Si}}\left(\mathrm{k}_{\|}= \pm 1.1 \AA^{-1}\right)$ strongly differ in effective mass and binding energy. The curvature of the band at $\overline{\mathrm{K}}_{\mathrm{Si}}$ corresponds to the one of the $\mathrm{S} 1$ surface state of a $(\sqrt{3} \times \sqrt{3}) \mathrm{R} 30^{\circ}-\mathrm{Ag}$ terminated $\mathrm{Si}(111)$ film. The intensity and binding energy of the bottom of this parabolic band is known to critically depend on the number of excess $\mathrm{Ag}$ atoms per $\mathrm{Si}(111)$ unit cell. ${ }^{58}$ This S1 band is barely visible in Fig. 6(a), indicating an almost stoichiometric $(\sqrt{3} \times \sqrt{3}) \mathrm{R} 30^{\circ}-\mathrm{Ag}$ surface composition, but can be identified easily (image with higher contrast) at the corresponding wave vector in the Fermi energy cut (Fig. 6(c)). The Fermi energy cuts (Fig. 6 (c, d)) also display a close resemblance of the Si film on $\operatorname{Ag}(111)$ and the $(\sqrt{3} \times \sqrt{3}) \mathrm{R} 30^{\circ}-\mathrm{Ag}$ $\mathrm{Si}(111)$ surface. The open-circle and point-like shapes at $\overline{\mathrm{K}}_{\mathrm{Si}}\left(\mathrm{k}_{\|}= \pm 1.1 \AA^{-1}\right)$ indicate that the $\mathrm{Ag}$ surface doping is slightly different in the two cases. In summary, the band at $\bar{\Gamma}_{\mathrm{Si}}\left(\mathrm{k}_{\|}=0 \AA^{-1}\right)$ originates from the $\mathrm{Si}-\mathrm{Ag}$ interface state, while the band at $\overline{\mathrm{K}}_{\mathrm{Si}}\left(\mathrm{k}_{\|}= \pm 1.1 \AA^{-1}\right)$ reproduces the $\mathrm{S} 1$ surface state of a Ag-terminated $(\sqrt{3} \times \sqrt{3}) \mathrm{R} 30^{\circ}-\mathrm{Si}(111)$ film. The overall band structure of multilayer Si film on $\mathrm{Ag}(111)$ closely correspond to $(\sqrt{3} \times \sqrt{3}) \mathrm{R} 30^{\circ}-\mathrm{Ag}$ surface terminated $\mathrm{Si}(111)$, with no evidence for multilayer silicene and for Dirac cones. 


\section{CONCLUSIONS}

LEED, LEEM, core level and angle resolved photoemission were employed to examine $\mathrm{Si}$ films on $\mathrm{Ag}(111)$, in order to assess whether monolayer and multilayer silicene is formed with graphene-like properties. Photoemission measurements on a honeycomb $(4 \times 4)$-silicene show that bands previously attributed to a Dirac-like $\pi$-silicene state derive from Ag bulk states and from interface states with saddle topology. The electronic structure of multilayer Si films on $\operatorname{Ag}(111)$ is found to closely correspond to that of $(\sqrt{3} \times \sqrt{3}) \mathrm{R} 30^{\circ}-\mathrm{Ag}$ segregated $s p^{3}$ diamond-like $\mathrm{Si}$. Photoemission results find that the bands previously attributed to $\pi^{*}$ state with Dirac fermion character are derived from the $\mathrm{Ag}$ induced $(\sqrt{3} \times \sqrt{3}) \mathrm{R} 30^{\circ}-\mathrm{Si}(111)$ surface states and from a Simodified $\mathrm{Ag}(111)$ interface state. 


\section{References}

${ }^{1}$ K. S. Novoselov, A. K. Geim, S. V. Morosov, D. Jiang, Y. Zhang, S. V. Dubonos, I. V. Grigorieva and A. A. Firsov, Science 306, 666 (2004).

2 J. C. Meyer, A. K. Geim, M. I. Katsnelson, K. S. Novoselov, T. J. Booth and S. Roth, Nature 446, 60 (2007).

${ }^{3}$ M. I. Katsnelson, K.S. Novoselov and A. K. Geim, Nature Phys. 2, 620 (2006).

${ }^{4}$ A. H. Castro Neto, F. Guinea, N. M. R. Peres, K. S. Novoselov, and A. K. Geim, Rev. Mod. Phys. 81, 109 (2009)

${ }^{5}$ S. Cahangirov, M. Topsakal, E. Aktrk, H. Sahin, and S. Ciraci, Phys. Rev. Lett. 102, 236804 (2009).

${ }^{6}$ K. Takeda and K. Shiraishi, Phys. Rev. B 50, 14916 (1994).

${ }^{7}$ G. G. Guzman-Verri and L. C. Lew Yan Voon, Phys. Rev. B 76, 075131 (2007).

${ }^{8}$ S. Lebgue and O. Eriksson, Phys. Rev. B 79, 115409 (2009).

${ }^{9}$ S. Huang, W. Kang, and L. Yang, Appl. Phys. Lett. 102, 133106 (2013).

${ }^{10}$ L. Tao, E. Cinquanta, D. Chiappe, C. Grazianetti, M. Fanciulli, M. Dubey, A. Molle and D. Akinwande, Nature Nanotech. 10, 227 (2015).

${ }^{11}$ B. Lalmi, H. Oughaddou, H. Enriquez, A. Kara, S.Vizzini, B. Ealet, and B. Aufray, Appl. Phys. Lett. 97, 223109 (2010).

${ }^{12}$ H. Jamgotchian, Y. Colignon, N. Hamzaoui, B. Ealet, J. Y. Hoarau, B. Aufray, and J. P. Bibrian, J. Phys.: Condens. Matter 24, 172001 (2012).

${ }^{13}$ C. L. Lin, R. Arafune, K. Kawahara, N. Tsukahara, E. Minamitani, Y. Kim, N. Takagi, and M. Kawai, Appl. Phys. Express 5, 045802 (2012).

${ }^{14}$ D. Chiappe, C. Grazianetti, G. Tallarida, M. Fanciulli, and A. Molle, Adv. Mater. 24, 5088 (2012).

${ }^{15}$ B. Feng, Z. J. Ding, S. Meng, Y. G. Yao, X. Y. He, P. Cheng, L. Chen, and K. H. Wu, Nano Lett. 12, 3507 (2012).

${ }^{16}$ P. Vogt, P. De Padova, C. Quaresima, J. Avila, E. Frantzeskakis, M. C. Asensio, A. Resta, B. Ealet, and G. Le Lay, Phys. Rev. Lett. 108, 155501 (2012).

${ }^{17}$ L. Chen, C. C. Liu, B. Feng, X. He, P. Cheng, Z. Ding, S. Meng, Y. G. Yao, and K.H. Wu, Phys. Rev. Lett. 109, 056804 (2012).

${ }^{18}$ R. Arafune, C. Lin, K. Kawahara, N. Tsukahara, E. Minamitani, Y. Kim, N. Takagi, and M. Kawai, Surf. Sci. 608, 297 (2013).

${ }^{19}$ L. Chen, H. Li, B. Feng, Z. Ding, J. Qiu, P. Cheng, K. Wu, and S. Meng, Phys. Rev. Lett. 110, 085504 (2013). 
${ }^{20}$ L. Chen, B. Feng, and K. Wu, Appl. Phys. Lett. 102, 081602 (2013).

${ }^{21}$ C. L. Lin, R. Arafune, K. Kawahara, M. Kanno, N. Tsukahara, E. Minamitani, Y. Kim, M. Kawai, and N. Takagi, Phys. Rev. Lett. 110, 076801 (2013). ${ }^{22}$ A. Fleurence, R. Friedlein, T. Ozaki, H. Kawai, Y.Wang, and Y. Yamada-Takamura, Phys. Rev. Lett. 108, 245501 (2012).

${ }^{23}$ R. Friedlein, A. Fleurence, J. T. Sadowski, and Y. Yamada-Takamura, Appl. Phys. Lett. 102, 221603 (2013).

${ }^{24}$ L. Meng, Y. Wang, L. Zhang, S. Du, R. Wu, L. Li, Y. Zhang, G. Li, H. Zhou, W. A. Hofer, and H.-J. Gao, Nano Lett. 13, 685 (2013).

${ }^{25}$ M. Satta, S. Colonna, R. Flammini, A. Cricenti, and F. Ronci, Phys. Rev. Lett. 115026102 (2015).

${ }^{26}$ P. Moras, T. O. Mentes, P. M. Sheverdyaeva, A. Locatelli, and C. Carbone, J. Phys.: Condens. Matter, 26, 185001 (2014).

${ }^{27}$ P. De Padova, J. Avila, A. Resta, I.Razado-Colambo, C. Quaresima, C. Ottaviani, B. Olivieri, T. Bruhn, P. Vogt, M.C.Asensio and G. Le Lay, J. Phys.: Condens. Matter 25382202 (2013).

${ }^{28}$ J. Avila, P. De Padova, S. Cho, I. Colambo, S. Lorcy, C. Quaresima, P. Vogt, A. Resta, G. Le Lay, and M. C. Asensio, J. Phys.: Condens. Matter 25, 262001 (2013).

${ }^{29}$ Z.-X. Guo, S. Furuya, J. Iwata and, A. Oshiyama, Phys. Rev. B 87, 235435 (2013).

${ }^{30}$ Y.-P. Wang and H.-P. Cheng, Phys. Rev. B 87, 245430 (2013).

${ }^{31}$ S. Cahangirov, M. Audffred, P. Tang, A. Iacomino, W. Duan, G. Merino and A. Rubio, Phys. Rev. B. 88, 035432 (2013).

${ }^{32}$ P. De Padova, P. Vogt, A. Resta, J. Avila, I. Razado-Colambo, C. Quaresima, C. Ottaviani, B. Olivieri, T. Bruhn, T. Hirahara, T. Shirai, S. Hasegawa, M. C. Asensio and G. Le Lay, Appl. Phys. Lett. 102, 163106 (2013).

${ }^{33}$ P. Vogt, P. Capiod, M. Berthe, A. Resta, P. De Padova, T. Bruhn, G. Le Lay and B. Grandidier, Appl. Phys. Lett. 104, 021602 (2014).

${ }^{34}$ E. Salomon, R. El Ajjouri, G. Le Lay and T. Angot, J. Phys.: Condens. Matter 26185003 (2014).

${ }^{35}$ N. W. Johnson, D. Muir, E. Z. Kurmaev and A. Moewes, Adv. Funct. Mater. 25, 4083 (2015).

${ }^{36}$ S. Cahangirov, V. O. Özçelik, A. Rubio, and S. Ciraci, Phys. Rev. B 90, 085426 (2014).

${ }^{37}$ A. J. Mannix, B. Kiraly, B. L. Fisher, M. C. Hersam and N. P. Guisinger, ACS Nano, 8, 7538 (2014).

${ }^{38}$ Y. Borensztein, A. Curcella, S. Royer and G. Prévot, Phys. Rev. B 92, 155407 (2015).

${ }^{39}$ G. Prévot, R. Bernard, H. Cruguel and Y. Borensztein, Appl. Phys. Lett. 105, 213106 (2014).

${ }^{40}$ T. Yamagami, J. Sone, K. Nakatsuji and H. Hirayama, Appl. Phys. Lett. 105, 151603 (2014). 
${ }^{41}$ J. Chen, Y. Du, Z. Li, W. Li, B. Feng, J. Qiu, P. Cheng, S. X. Dou, L. Chen and K. Wu, Sci. Rep. 5, 13590 (2015).

${ }^{42}$ Z.-X. Guo and A. Oshiyama, New J. Phys. 17, 045028 (2015).

${ }^{43}$ G.-W. Lee, H.-D. Chen, D.-S. Lin, Appl. Surf. Sci. 354, 212 (2015).

${ }^{44}$ K. Kawahara, T. Shirasawa, C.-L. Lin, R. Nagao, N. Tsukahara, T. Takahashi, R. Arafune, M. Kawai, N. Takagi, Surf. Sci. 651, 70 (2016).

45 T. Shirai, T. Shirasawa, T. Hirahara, N. Fukui, T. Takahashi and S. Hasegawa, Phys. Rev. B 89, 241403(R) (2014).

${ }^{46}$ T. O. Menteş, G. Zamborlini, A. Sala and A. Locatelli, Beilstein J. Nanotechnol. 5, 1873 (2014).

${ }^{47}$ G. Kresse and J. Furthmueller, Phys. Rev. B 54, 11169 (1996); G. Kresse and D. Joubert, Phys. Rev. B 59, 1758 (1999).

48 J. P. Perdew, K. Burke, and M. Ernzerhof, Phys. Rev. Lett. 77, 3865 (1996).

${ }^{49}$ S. Grimme, J. Comput. Chem. 27, 1787 (2006).

${ }^{50}$ S. K. Mahatha, P. Moras, V. Bellini, P. M. Sheverdyaeva, C. Struzzi, L. Petaccia, and C. Carbone, Phys. Rev. B 89, 201416(R) (2014).

${ }^{51}$ D. Tsoutsou, E. Xenoginnopoulou, E. Golias, P. Tsipas and A Dimoulas, Appl. Phys. Lett. 103, 231604 (2013).

${ }^{52}$ H. Ishida, Y. Hamamoto, Y. Morikawa, E. Minamitani, R. Arafune and N. Takagi, New J. Phys. 17, 015013 (2015).

${ }^{53}$ S. K. Mahatha, P. Moras, P. M. Sheverdyaeva, R. Flammini, K. Horn and C. Carbone, Phys. Rev. B 92, 245127 (2015).

${ }^{54}$ J. A. Adams, A. Bostwick, T. Ohta, F. S. Ohuchi and M. A. Olmstead, Phys. Rev. B 75, 195308 (2005).

${ }^{55}$ F. Reinert, G. Nicolay, S. Schmidt, D. Ehm, and S. Hüfner, Phys. Rev. B 63, 115415 (2001).

${ }^{56}$ X. Tong, S. Ohuchi, N. Sato, T. Tanikawa, T. Nagao, I. Matsuda, Y. Aoyagi and S. Hasegawa, Phys. Rev. B 64, 205316 (2001).

${ }^{57}$ L. S. O. Johansson, E. Landemark, C. J. Karlsson and R. I. G. Uhrberg, Phys. Rev. Lett.63, 2092 (1989).

${ }^{58}$ J. N. Crain, M. C. Gallagher, J. L. McChesney, M. Bissen and F. J. Himpsel, Phys. Rev. B 72, 045312 (2005). 


\section{Figure captions}

Figure 1: Si growth on $\mathrm{Ag}(111)$ at $\sim 500 \mathrm{~K}$ observed by LEED at $31 \mathrm{eV}$ electron energy. (a) Clean Ag surface; (b) $0.5 \mathrm{ML}$, (c) $1.0 \mathrm{ML}$, (d) $1.4 \mathrm{ML} \mathrm{Si}$ on $\mathrm{Ag}(111)$. The $(\sqrt{3} \times \sqrt{3}) \mathrm{R} 30^{\circ}$ phase resulting from the second layer of a Si film is marked with a dashed hexagon.

Figure 2: (a) LEED for $1 \mathrm{ML} \mathrm{Si} / \mathrm{Ag}(111)$ grown at $500 \mathrm{~K}$ with electron primary energy $31 \mathrm{eV}$. (b) Sum of the two dark-field LEEM images obtained using the $(\sqrt{13} \times \sqrt{13}) \mathrm{R} 13.9^{\circ}$ spots. The red contours mark the domain boundaries. The area coverage of the $(4 \times 4)$ structure is $\sim 43 \%$. (c) Surface Brillouin zones of $\operatorname{Ag}(111)$ (black line), (1×1) silicene (green line) and (4×4) silicene (red line) and $(\sqrt{3} \times \sqrt{3}) \mathrm{R} 30^{\circ}$ silicene (blue line). Red and blue dots indicate high symmetry points of the $(4 \times 4)$ and $(\sqrt{3} \times \sqrt{3}) \mathrm{R} 30^{\circ}$ cells respectively.

Figure 3: (a) Calculated band dispersion of $\sigma$ (green) and $\pi$ (orange) states of a free-standing $(1 \times 1)$ silicene layer. (b) Angle-resolved photoemission spectra of the $(4 \times 4)$ silicene grown on $\operatorname{Ag}(111)$ using a photon energy of $135 \mathrm{eV}$. Dashed green lines follow the dispersion of the Si- $\sigma$ state and the white box shows the area of interest zoomed in Fig 4.

Figure 4: Angle-resolved photoemission spectra of (a, b) silicene/Ag(111) and (c, d) clean $\operatorname{Ag}(111)$ along $\bar{\Gamma}_{\mathrm{Ag}}-\overline{\mathrm{K}}_{\mathrm{Ag}}$ measured using photon energy of $126 \mathrm{eV}$. Angle-resolved photoemission spectra for (e) silicene/Ag(111) surface and (g) clean $\operatorname{Ag}(111)$ along $\bar{\Gamma}_{\mathrm{Ag}}-\overline{\mathrm{M}}_{\mathrm{Ag}}$ measured using photon energy of $135 \mathrm{eV}$ and along its (f, g) orthogonal $\overline{\mathrm{K}}_{\mathrm{Ag}}-\overline{\mathrm{M}}_{\mathrm{Ag}}-\overline{\mathrm{K}}_{\mathrm{Ag}}$ directions respectively. (i) Sketch of a saddle structure with maxima along $\bar{\Gamma}_{\mathrm{Ag}}-\overline{\mathrm{M}}_{\mathrm{Ag}}-\bar{\Gamma}_{\mathrm{Ag}}$ direction and minima along orthogonal $\overline{\mathrm{K}}_{\mathrm{Ag}}-\overline{\mathrm{M}}_{\mathrm{Ag}}-\overline{\mathrm{K}}_{\mathrm{Ag}}$ direction.

Figure 5: (a) $\mathrm{Ag} 3 d_{5 / 2}$ core level spectra of $7 \mathrm{ML} \mathrm{Si}$ on $\mathrm{Ag}(111)$ with decomposed components. Area under the $\mathrm{Ag} 3 d_{5 / 2}$ peak as a function of thickness is shown in inset. (b) Si $2 p$ core level spectra of a $7 \mathrm{ML}$ Si film on $\mathrm{Ag}(111)$ (red line) and $(\sqrt{3} \times \sqrt{3}) \mathrm{R} 30^{\circ}-\mathrm{Ag}$ on $\mathrm{Si}(111)$ (black line). (c) $\mathrm{Ag} 3 d_{5 / 2}$ core level spectra of clean $\mathrm{Ag}(111)$ (black line), Si-Ag step edge (red line) and thick $\mathrm{Si}$ film (blue line) using $430 \mathrm{eV}$ photon energy. (d) The real picture of a thick Si film (purple) deposited on $\operatorname{Ag}(111)$ surface, marked with illumination areas on different parts of the sample by yellow dots. 
Figure 6: Angle-resolved photoemission spectra (first derivative) of (a) multilayer $\mathrm{Si} / \mathrm{Ag}(111)$ and (b) $(\sqrt{3} \times \sqrt{3}) \mathrm{R} 30^{\circ}-\mathrm{Ag}$ on $\mathrm{Si}(111)$ surface, along the $\overline{\mathrm{K}}_{\mathrm{Si}}-\bar{\Gamma}_{\mathrm{Si}}-\overline{\mathrm{M}}_{\mathrm{Si}}$ symmetry direction measured using a photon energy of $126 \mathrm{eV}$. Fermi energy cuts of (c) multilayer $\mathrm{Si} / \mathrm{Ag}(111)$ and (d) $(\sqrt{3} \times \sqrt{3}) \mathrm{R} 30^{\circ}-\mathrm{Ag}$ on $\mathrm{Si}(111)$ surface. Red parabola indicates the Ag L-gap interface state (I), while white arrows indicate surface states of $(\sqrt{3} \times \sqrt{3}) \mathrm{R} 30^{\circ}-\mathrm{Ag}$ on $\mathrm{Si}(111)$ surface. Surface Brillouin zones of $(1 \times 1)$-Si and $(\sqrt{3} \times \sqrt{3}) \mathrm{R} 30^{\circ}$-Si are shown as green and blue hexagons respectively. 


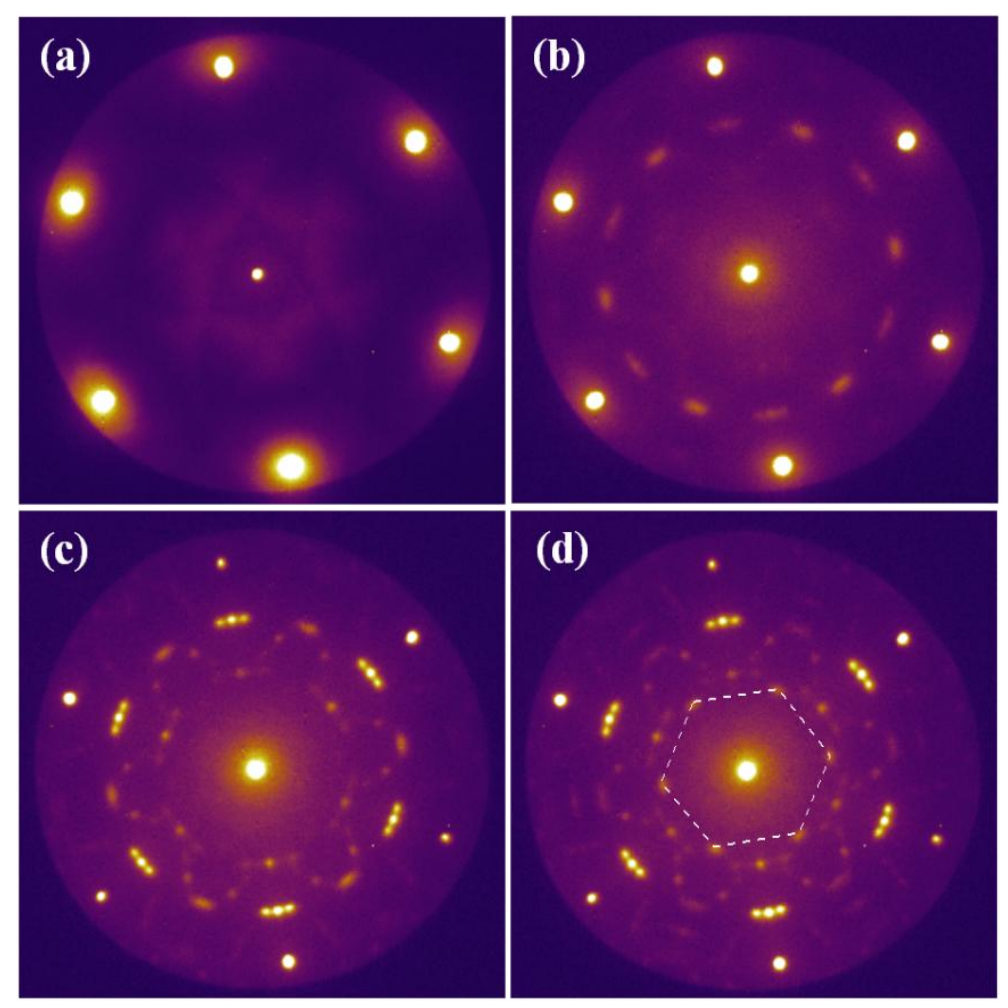

Figure 1 

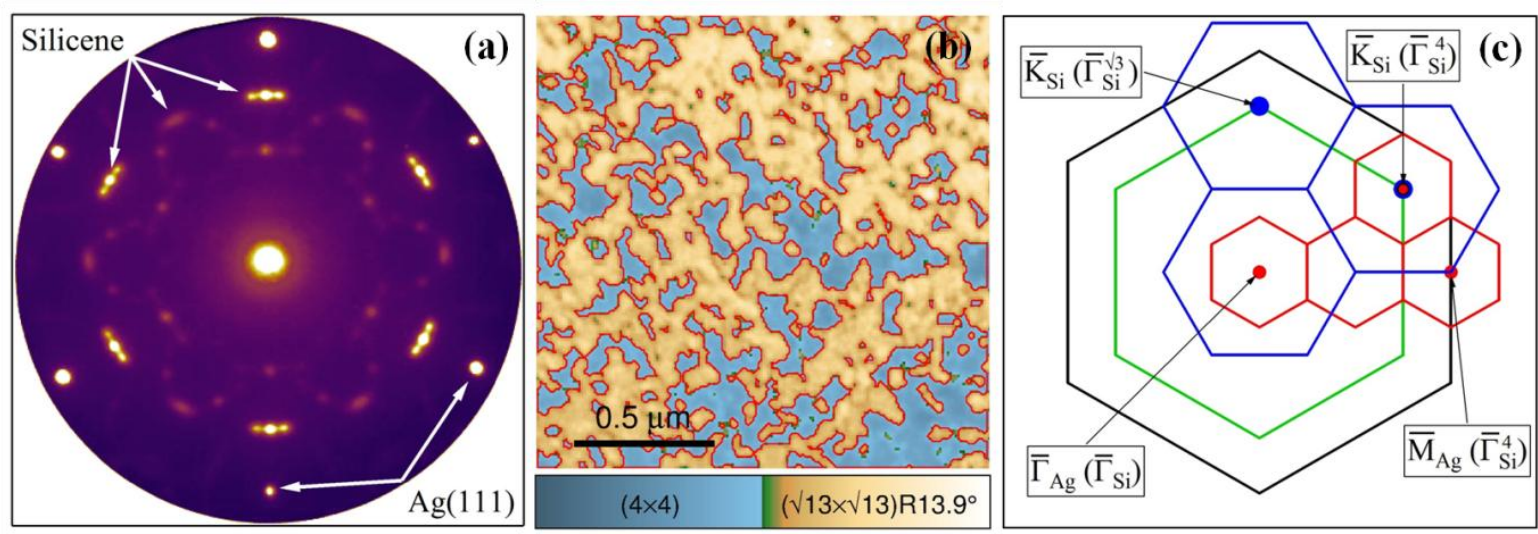

Figure 2 


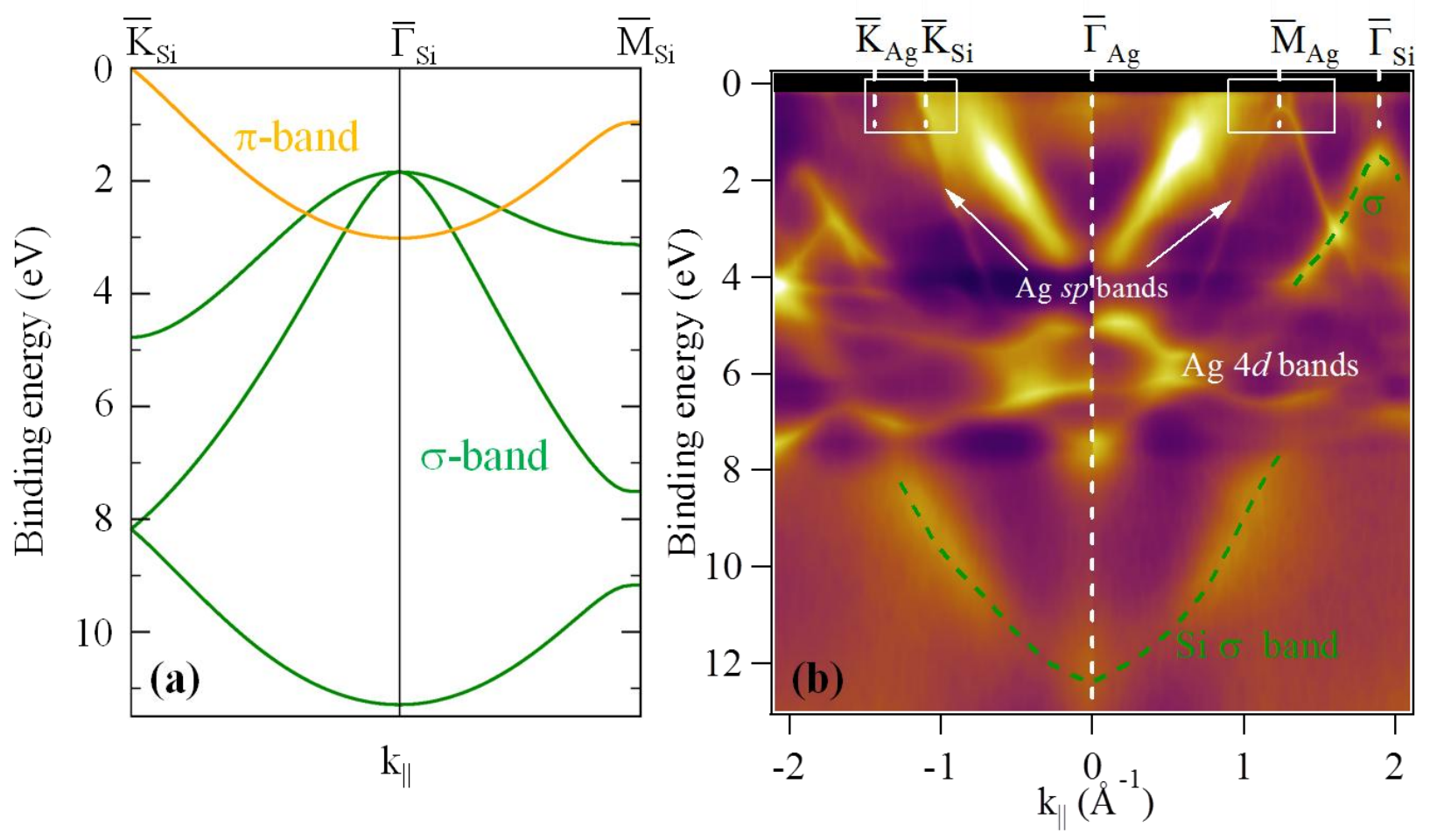

Figure 3 


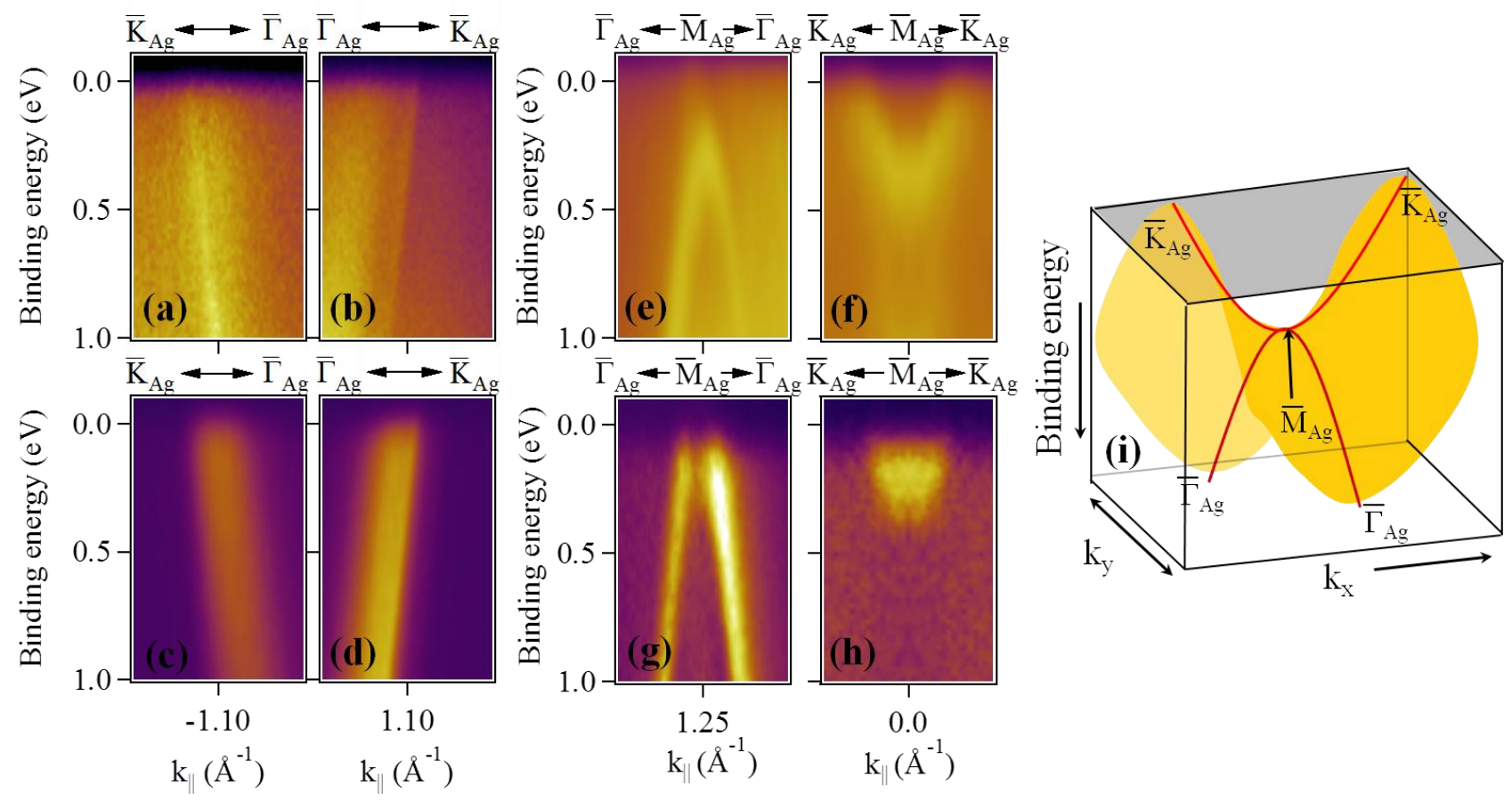

Figure 4 

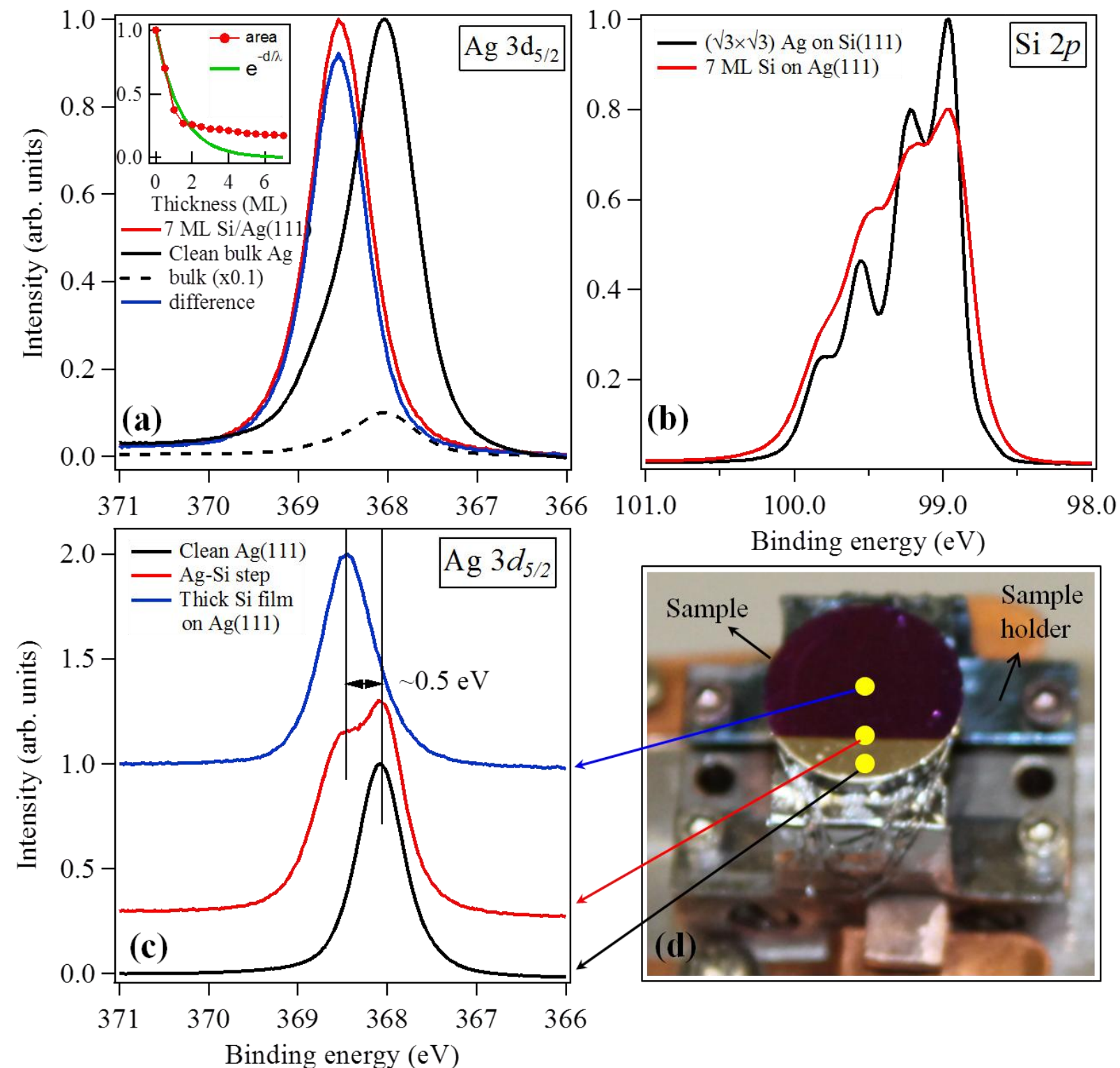

Figure 5 

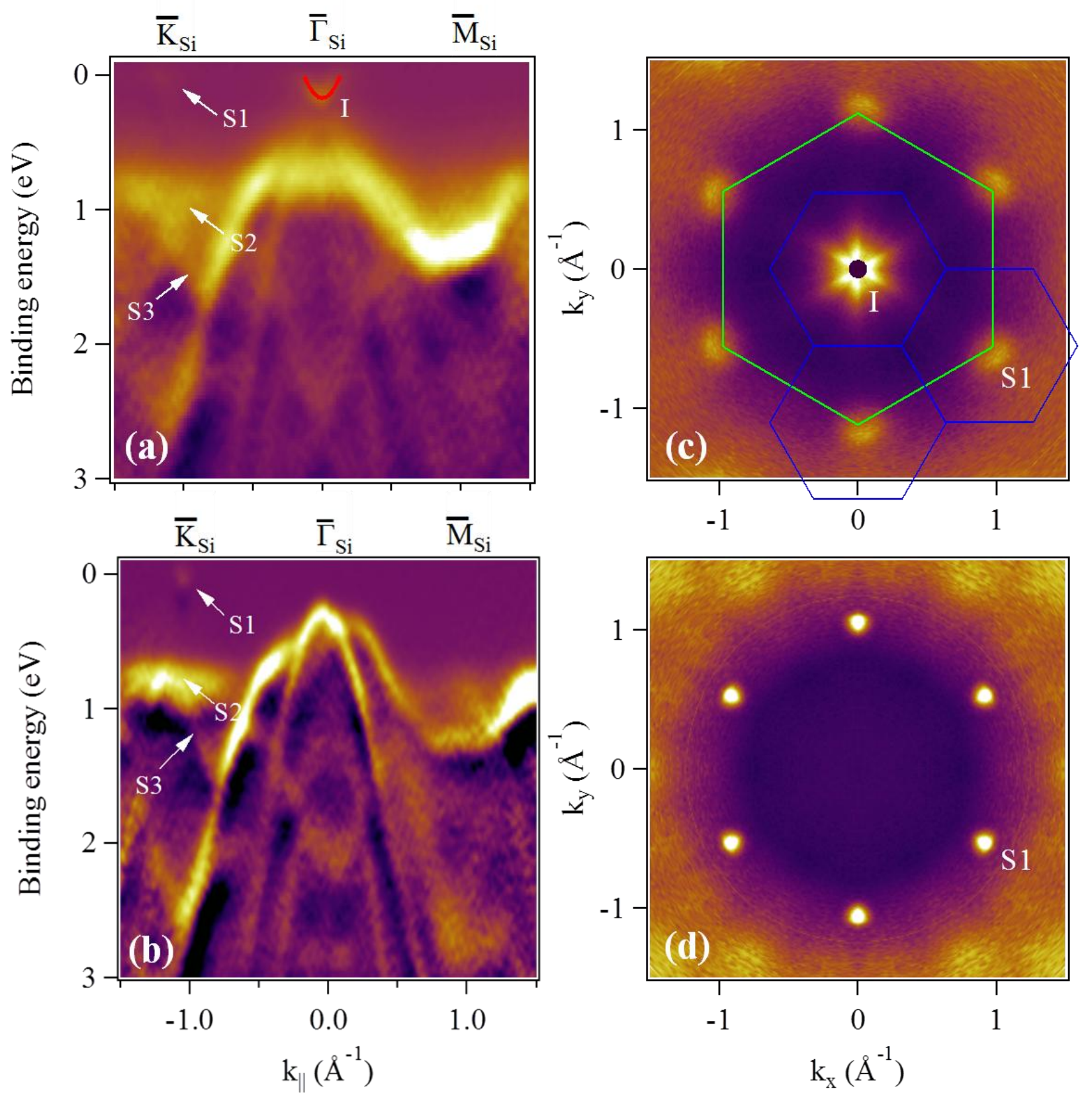

Figure 6 